\title{
Heart Rate-corrected QT Interval Duration in Rheumatoid Arthritis and Its Reduction with Treatment with the Interleukin 6 Inhibitor Tocilizumab
}

\author{
Hitomi Kobayashi, Yasuyuki Kobayashi, Isamu Yokoe, Noboru Kitamura, Atsuma Nishiwaki, \\ Masami Takei, and Jon T. Giles
}

ABSTRACT. Objective. Individuals with rheumatoid arthritis (RA) are at a heightened risk of sudden cardiac death, an outcome increased in those with prolongation of the corrected electrocardiographic QT interval (QTc). We compared QTc between patients with RA and demographically matched controls and studied the change in QTc after treatment with the interleukin 6 inhibitor tocilizumab (TCZ).

Methods. Standard 12-lead electrocardiograms were obtained and QTc was measured in patients with RA at baseline and after 24 weeks of TCZ treatment, then compared with non-RA controls who were frequency-matched on age and sex. Indicators of the baseline QTc and predictors of change in QTc were studied using multivariable linear regression.

Results. A total of 94 RA and 42 non-RA controls were studied. The average baseline QTc was $10 \mathrm{~ms}$ longer in the RA group vs controls (422 vs $412 \mathrm{~ms}$, respectively; $\mathrm{p}<0.001$ ) and decreased to an average of $406 \mathrm{~ms}$ with treatment $(\mathrm{p}<0.001)$. Baseline QTc was significantly and independently higher among those with anticyclic citrullinated peptide antibodies seropositivity, higher swollen joint counts, and higher levels of C-reactive protein (CRP) and matrix metalloproteinase 3. Each log unit decrease in CRP with treatment was associated with an average reduction in QTc of $2.9 \mathrm{~ms}(\mathrm{p}=0.002)$ after adjusting for age and baseline QTc. Clinical response measures were not associated with the change in QTc.

Conclusion. The marked normalization of QTc observed with TCZ treatment, and its close parallel with CRP reduction, support the premise that systemic inflammation contributes to cardiac repolarization abnormalities in RA that may be amenable to treatment. (First Release September 12018 ; J Rheumatol 2018;45:1620-7; doi:10.3899/jrheum.180065)

\section{Key Indexing Terms: \\ RHEUMATOID ARTHRITIS \\ CARDIAC ELECTROPHYSIOLOGY}

Individuals with rheumatoid arthritis (RA) have a 2-fold higher risk of sudden cardiac death compared with age- and sex-matched controls ${ }^{1}$. Among the general (i.e., non-RA) population, several large studies have established that the risk

From the Division of Hematology and Rheumatology, Nihon University School of Medicine, Tokyo; Department of Advanced Biomedical Imaging Informatics, St. Marianna University School of Medicine, Kawasaki, Japan; Division of Rheumatology, Columbia University, College of Physicians and Surgeons, New York, New York, USA.

H. Kobayashi, MD, PhD, Division of Hematology and Rheumatology, Nihon University School of Medicine; Y. Kobayashi, $M D, P h D$,

Department of Advanced Biomedical Imaging Informatics, St. Marianna

University School of Medicine; I. Yokoe, MD, Division of Hematology and Rheumatology, Nihon University School of Medicine; N. Kitamura, MD, Division of Hematology and Rheumatology, Nihon University School of Medicine; A. Nishiwaki, MD, Division of Hematology and Rheumatology, Nihon University School of Medicine; M. Takei, MD, PhD, Division of Hematology and Rheumatology, Nihon University School of Medicine; J.T. Giles, MD, MPH, Division of Rheumatology, Columbia University, College of Physicians and Surgeons.

Address correspondence to Dr. J.T. Giles, Division of Rheumatology, Columbia University, College of Physicians and Surgeons, 630 West 168th St., P\&S Building, 3rd Floor, Suite 3450, New York, New York 10032, USA. E-mail: jtg2122@cumc.columbia.edu

Accepted for publication May 28, 2018.

\section{CARDIOVASCULAR DISEASE} INFLAMMATION of sudden cardiac death is increased in individuals with prolongation of the corrected QT (QTc) interval, as measured with electrocardiography $(\mathrm{ECG})^{2,3}$. Chauhan, et al reported a significantly elevated risk of developing QTc prolongation among patients with $\mathrm{RA}^{4}$, and as reported by Panaoulas, et $a l$, each $50 \mathrm{~ms}$ longer QTc was associated with a more than 2 -fold higher hazard of all-cause mortality over an average followup time of 6 years 5 .

In many of these studies, circulating C-reactive protein $(\mathrm{CRP})^{4,6}$ and/or circulating inflammatory cytokines ${ }^{7}$ were correlated with longer QTc intervals. These findings raise the possibility that RA treatments that reduce circulating levels of these offending cytokines may reduce the incidence of cardiac repolarization abnormalities that contribute to sudden cardiac death. However, whether QTc prolongation is reduced with treatment has not yet been well established. In a study of 17 patients with RA, Lazzerini, et al reported QTc normalization after treatment with the interleukin 6 (IL-6) inhibitor tocilizumab (TCZ), an effect that was correlated with the degree of reduction in $\mathrm{CRP}^{8}$. However, that study did not include comparison with a non-RA control group, and

Personal non-commercial use only. The Journal of Rheumatology Copyright @ $\odot$ 2018. All rights reserved 
with the small sample size, only simple correlations of characteristics with QTc were possible. We sought to examine the QTc interval in a larger group of patients with RA compared with a demographically matched group of non-RA controls. Additionally, we sought to study the independent inflammatory and noninflammatory indicators of QTc in patients with RA and characterize the predictors of change in QTc associated with 24 weeks of treatment with TCZ. We hypothesized that clinical response to TCZ would be associated with a reduction in QTc in patients with RA, potentially returning to levels comparable to those of individuals without RA.

\section{MATERIALS AND METHODS}

Study population. Patients with RA were recruited from the Itabashi Chuo Medical Center from March 2012 to May 2016. Patients met the 2010 American College of Rheumatology/European League Against Rheumatism (EULAR) criteria for RA ${ }^{9}$, had moderate to severe RA disease activity based on the 28-joint count Disease Activity Score (DAS28) cutpoints $^{10}$, and complied with the Guideline for Biological Products of the Japanese College of Rheumatology. Healthy volunteers were recruited as a control group to be frequency-matched to the age and sex distribution of the RA group. Both patients and controls were excluded if they reported prior cardiovascular (CV) events or procedures, whether self-reported or physician-diagnosed, including myocardial infarction, angioplasty, chronic heart failure, or arrhythmia. ECG exclusions included tachycardia (i.e., heart rate $>100$ bpm), left bundle branch block, and ST elevations or depressions. The study was approved by the local ethics committee (Nihon University Itabashi Hospital RK-170912-12), and informed consent was obtained from each patient in accordance with the Helsinki Declaration of 1975 (revised in 1983).

Assessment and treatment protocol. Patients received TCZ either once a month ( $8 \mathrm{mg} / \mathrm{kg}$ intravenously) or $162 \mathrm{mg}$ subcutaneously biweekly for 24 weeks. Electrocardiogram, clinical assessments, and laboratory monitoring were performed at baseline and Week 24 . All patients were clinically evaluated by the same observer for disease activity measures. Low disease activity (LDA) was achieved if the DAS28 using CRP (DAS28-CRP) was $<3.2$ units, and remission was achieved if the DAS28-CRP was $<2.6$ units $^{11}$. A EULAR good response was achieved if the DAS28-CRP achieved was $<3.2$ units and the change from baseline was $\geq 1.2$ units $^{11}$.

ECG assessment. Resting 12-lead ECG $(25 \mathrm{~mm} / \mathrm{s}$ paper speed and 10 $\mathrm{mm} / \mathrm{mV}$ amplitude) were recorded using a 3-channel direct writing machine at baseline and Week 24. The QT interval was corrected with the Bazett formula. A QTc $\geq 440 \mathrm{~ms}$ was considered prolonged, because this threshold has been demonstrated in several large studies to be associated with an increased risk of sudden cardiac death ${ }^{12,13}$. Accordingly, in this study the QTc interval of $440 \mathrm{~ms}$ was considered prolonged.

Clinical and laboratory assessments. Fasting samples of serum and plasma were collected at baseline and at Week 24, separated by centrifugation, and stored at $-70^{\circ} \mathrm{C}$. All assays were performed at our institution using our internal quality control procedures. At the time of the study visit, all subjects underwent routine laboratory investigations, and basic screening for traditional atherosclerotic disease risk factors, including history of cigarette smoking, serum cholesterol, triglycerides, high-density lipoprotein, low-density lipoprotein, fasting blood glucose concentration, rheumatoid factor, and anticyclic citrullinated peptide antibodies (anti-CCP). Collected samples were assessed blinded to group status or clinical characteristics. Matrix metalloproteinase (MMP-3) level was measured by the blood sample reacting with mouse antihuman MMP-3 monoclonal antibody in a turbidimetric immunoassay with sensitized latex-enhanced particles. CRP was measured by latex agglutination turbidimetric immunoassay.
Statistical analysis. Differences in participant characteristics according to disease status were compared using $\mathrm{t}$ tests for normally distributed continuous variables, the Kruskal-Wallis test for non-normally distributed continuous variables, and the chi-square goodness-of-fit test or Fisher's exact test, as appropriate, for categorical variables. Linear regression was used to model predictors of baseline and change in QTc interval. Predictors were first modeled separately, and those with an association $p$ value $<0.20$ were carried into an extended multivariable (MV) model. Noncontributory covariates were excluded using Akaike Information Criterion for nested models. The adjusted $\mathrm{R}^{2}$ was used to assess the proportion of the variability in the outcome explained by the modeled covariates. The Shapiro-Wilk test was used to assess that normality assumptions for linear regression were met, which was the case for all the final MV models. Variance inflation factors were calculated to assess for collinearity among modeled covariates, and none was detected in all models. Statistical interaction of change in CRP with baseline QTc was tested in linear regression by introducing a QTc $\times$ change in CRP interaction term into the model. All statistical calculations were performed using Intercooled Stata 14 (StataCorp). A 2-tailed $\alpha$ of 0.05 was used throughout.

\section{RESULTS}

Baseline (pretreatment) characteristics of the 94 patients with RA and 42 non-RA controls are summarized in Table 1. The groups did not significantly differ on age or sex. Three-quarters of the patients had no CV disease (CVD) risk factors. Among those with CVD risk factors, the majority had hypertension $(\mathrm{n}=14)$. Hyperlipidemia was present in 6 patients with RA, and only 1 had diabetes. RA disease activity was moderate to high, on average, and was reflected in above-normal markers of systemic inflammation in most. The majority of patients with RA (81\%) were treated with disease-modifying antirheumatic drugs (DMARD), most prominently with methotrexate (MTX) at a median dose of $8 \mathrm{mg}$ per week. Other DMARD (bucillamine, tacrolimus, and sulfasalazine) were uncommon (15\%) and were used alone or in combination with MTX. Almost half were treated with prednisolone at baseline.

After 24 weeks of treatment, statistically significant improvements were observed in all disease activity measures, circulating inflammatory markers, and Health Assessment Questionnaire scores. Eighty-five percent achieved DAS LDA, $80 \%$ were in DAS remission, and $71 \%$ had no more than 1 swollen joint.

Elevated baseline QTC intervals decreased with TCZ treatment. The average baseline QTc was $10 \mathrm{~ms}$ longer in the RA group compared with controls (422 vs $412 \mathrm{~ms}$, respectively; $\mathrm{p}<0.001$ : Figure 1A). After treatment, the average QTc decreased to $406 \mathrm{~ms}$ in the RA group and was now significantly lower than the level of the control group. There were 12 RA patients with a baseline QTc $\geq 440 \mathrm{~ms}$ compared with none of the controls ( $p=0.018$, data not shown). After treatment, only 1 patient still had a QTc $\geq 440 \mathrm{~ms}$. Treatment-associated reduction in QTc to levels at or below those of non-RA controls was observed in both men and women with RA (Figure 1B).

Baseline QTC interval was associated with anti-CCP seropositivity and multiple indicators of disease activity. Univariate

Personal non-commercial use only. The Journal of Rheumatology Copyright $\subset$ 2018. All rights reserved. 
Table 1. Baseline and followup characteristics. Data are median (interquartile range) unless otherwise indicated.

\begin{tabular}{|c|c|c|c|}
\hline Characteristics & $\mathrm{RA}, \mathrm{n}=94$ & Controls, $\mathrm{n}=42$ & $\mathrm{p}$ \\
\hline Age, yrs, mean \pm SD & $56 \pm 11$ & $57 \pm 11$ & 0.71 \\
\hline Male, $\mathrm{n}(\%)$ & $18(19)$ & $10(24)$ & 0.54 \\
\hline Systolic blood pressure, $\mathrm{mm} \mathrm{Hg}$, mean $\pm \mathrm{SD}$ & $121 \pm 11$ & $120 \pm 7$ & 0.54 \\
\hline Diastolic blood pressure, $\mathrm{mm} \mathrm{Hg}$, mean $\pm \mathrm{SD}$ & $70 \pm 9$ & $70 \pm 6$ & 0.63 \\
\hline $\mathrm{HbA} 1 \mathrm{c}, \%$, mean $\pm \mathrm{SD}$ & $5.5 \pm 0.3$ & $5.5 \pm 0.3$ & 0.31 \\
\hline Total cholesterol, mg/dl & $187 \pm 26$ & $187 \pm 15$ & 0.95 \\
\hline LDL-C, $\mathrm{mg} / \mathrm{dl}$, mean $\pm \mathrm{SD}$ & $102 \pm 22$ & $101 \pm 13$ & 0.83 \\
\hline $\mathrm{HDL}-\mathrm{C}, \mathrm{mg} / \mathrm{dl}$, mean $\pm \mathrm{SD}$ & $63 \pm 15$ & $65 \pm 10$ & 0.34 \\
\hline Triglycerides, mg/dl & $116(92-133)$ & $101(90-120)$ & 0.11 \\
\hline RA duration, mos & $15(7-72)$ & & \\
\hline $\mathrm{RF}>40$ units, $\mathrm{n}(\%)$ & $59(63)$ & & \\
\hline anti-CCP $>20$ units, $\mathrm{n}(\%)$ & $67(71)$ & & \\
\hline DAS28, units, mean \pm SD & $4.9 \pm 1.2$ & & \\
\hline SJC & $4(2-6)$ & & \\
\hline TJC & $4(2-6)$ & & \\
\hline SDAI, units & $22.0(14.3-30.0)$ & & \\
\hline $\mathrm{CRP}, \mathrm{mg} / \mathrm{dl}$ & $2.0(0.8-3.4)$ & & \\
\hline $\mathrm{ESR}, \mathrm{mm} / \mathrm{h}$ & $34(20-52)$ & & \\
\hline MMP-3, ng/ml & $150(107-277)$ & & \\
\hline VAS, mm & $50(30-75)$ & & \\
\hline mVAS, mm & $58(40-70)$ & & \\
\hline HAQ, units & $0.25(0.12-0.94)$ & & \\
\hline Any DMARD, n (\%) & $76(81)$ & & \\
\hline MTX, n (\%) & $66(70)$ & & \\
\hline MTX dose, mg & $8(8-10)$ & & \\
\hline Other DMARD, n (\%) & $14(15)$ & & \\
\hline Prednisolone, $\mathrm{n}(\%)$ & $45(48)$ & & \\
\hline Prednisolone dose (users), mg/day & $5(4-5)$ & & \\
\hline Change in DAS28, units, mean \pm SD & $-2.93 \pm 1.31^{*}$ & & \\
\hline EULAR good response, $\mathrm{n}(\%)$ & $74(79)^{*}$ & & \\
\hline Low disease activity, $\mathrm{n}(\%)$ & $80(85)^{*}$ & & \\
\hline Remission, $\mathrm{n}(\%)$ & $75(80)^{*}$ & & \\
\hline $\mathrm{SJC} \leq 1$ joint, $\mathrm{n}(\%)$ & $67(71)^{*}$ & & \\
\hline$\%$ change, mean \pm SD & $-59 \pm 20 *$ & & \\
\hline Change in CRP, $\mathrm{mg} / \mathrm{dl}$ & $-2.0(-3.4 \text { to }-0.8)^{*}$ & & \\
\hline Change in ESR, $\mathrm{mm} / \mathrm{h}$ & $-24(-47 \text { to }-14)^{*}$ & & \\
\hline Change in MMP-3, ng/ml & $-85(-197 \text { to }-27)^{*}$ & & \\
\hline Change in VAS, mm & $-30(-50 \text { to }-10)^{*}$ & & \\
\hline Change in HAQ, units & $-0.12(-0.44 \text { to } 0)^{*}$ & & \\
\hline Change in prednisolone dose, $\mathrm{mg} /$ day & $-2(-4$ to -1$)$ & & \\
\hline Stopped prednisolone, $\mathrm{n}(\%)$ & $13(14)$ & & \\
\hline
\end{tabular}

* P value $<0.05$ for change between baseline and Week 24. RA: rheumatoid arthritis; HDL-C: high-density lipoprotein cholesterol; LDL-C: low-density lipoprotein cholesterol; RF: rheumatoid factor; anti-CCP: anticyclic citrullinated peptide; DAS28: 28-joint count Disease Activity Score; SDAI: Simplified Disease Activity Index; CRP: C-reactive protein; ESR: erythrocyte sedimentation rate; MMP: matrix metalloproteinase; VAS: visual analog scale; mVAS: modified VAS; DMARD: disease-modifying antirheumatic drug; HAQ: Health Assessment Questionnaire; MTX: methotrexate; EULAR: European League Against Rheumatism; SJC: swollen joint count; TJC: tender joint count.

and multivariable associations of RA patient characteristics with baseline QTc interval are summarized in Table 2. In the final model, those seropositive for anti-CCP had an adjusted QTc interval that was $8.3 \mathrm{~ms}$ longer than those who were seronegative $(\mathrm{p}=0.021)$. On average, each additional swollen joint was associated with a 1-ms longer adjusted QTc $(\mathrm{p}=0.038)$. Each log unit higher CRP was associated with a 3.7 ms longer adjusted QTc, on average, while each log unit higher MMP-3 was associated with a 5.2-ms longer adjusted
QTc. Together, these 4 variables accounted for $26 \%$ of the explainable variability in baseline QTc interval (i.e., $\left.\mathrm{R}^{2}=0.255 ; \mathrm{p}<0.001\right)$. Neither demographics, the presence of CVD risk factors, nor baseline use of DMARD or prednisolone were significantly associated with baseline QTc.

Patients above the median for swollen joint count (SJC; 4 joints), CRP (2.0 mg/dl), and MMP-3 (150 units) were classified as having the feature present. Those seropositive for anti-CCP and SJC, CRP, and MMP-3 all above the 

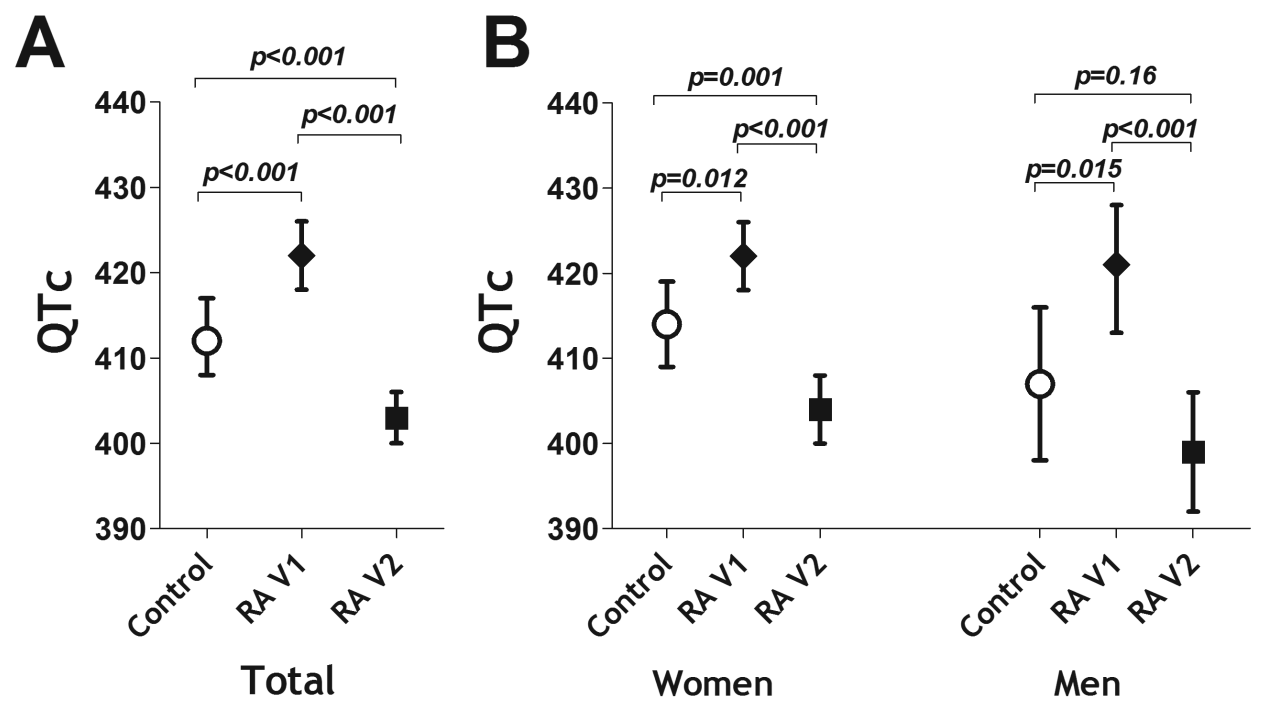

Figure 1. Comparison of QTc interval pre- and post-tocilizumab between RA patients and non-RA controls (baseline). Means and 95\% CI are depicted. QTc: corrected electrocardiographic QT interval; RA: rheumatoid arthritis.

Table 2. Associations of patient characteristics with baseline QTc interval.

\begin{tabular}{|c|c|c|c|c|c|c|}
\hline \multirow{2}{*}{ Variables } & \multicolumn{2}{|c|}{ Univariate Models } & \multicolumn{2}{|c|}{ Extended MV Model } & \multicolumn{2}{|c|}{ Reduced MV Model } \\
\hline & $\beta$ & $\mathrm{p}$ & $\beta$ & $\mathrm{p}$ & $\beta$ & $\mathrm{p}$ \\
\hline Age, per year & -0.12 & 0.48 & & & & \\
\hline Any CVD risk factors & -3.71 & 0.38 & & & & \\
\hline RA duration, per month & -0.0021 & 0.93 & & & & \\
\hline $\mathrm{RF}>40$ units & 5.75 & 0.13 & 1.16 & 0.74 & & \\
\hline SJC, per joint & 1.48 & 0.005 & 0.91 & 0.18 & 1.01 & 0.038 \\
\hline TJC, per joint & 0.67 & 0.090 & 0.091 & 0.85 & & \\
\hline Log SDAI, per unit & 1.89 & 0.60 & & & & \\
\hline Log CRP, per mg/dl & 5.30 & 0.001 & 3.74 & 0.016 & 3.69 & 0.016 \\
\hline$\sqrt{ } \mathrm{ESR}$, per $\mathrm{mm} / \mathrm{h}$ & 1.48 & 0.095 & & & & \\
\hline Log MMP-3, per ng/ml & 8.24 & $<0.001$ & 5.12 & 0.018 & 5.16 & 0.015 \\
\hline MTX & 1.24 & 0.76 & & & & \\
\hline MTX dose, mg & 0.42 & 0.32 & & & & \\
\hline Other DMARD & -0.39 & 0.94 & & & & \\
\hline Prednisolone & 1.28 & 0.73 & & & & \\
\hline Prednisolone dose, per mg/day & 0.81 & 0.21 & & & & \\
\hline Adjusted $\mathrm{R}^{2}$ & & & 0.239 & $<0.001$ & 0.255 & $<0.001$ \\
\hline
\end{tabular}

$\beta$ coefficients represent the average difference in baseline QTc interval per 1 unit higher of the indicated independent variable from linear regression modeling. The first column of univariate models represents individual models in which the indicated independent variable is the only covariate in the model. The MV models are single models containing each of the listed covariates. $\mathrm{R}^{2}$ represents the proportion of the variability of the baseline QTc interval explained by the covariates in the model. MV: multivariable; RA: rheumatoid arthritis; CVD: cardiovascular disease; RF: rheumatoid factor; anti-CCP: anticyclic citrullinated peptide; DAS28: 28-joint count Disease Activity Score; SJC: swollen joint count; TJC: tender joint count; SDAI: Simplified Disease Activity Index; CRP: C-reactive protein; ESR: erythrocyte sedimentation rate; MMP: matrix metalloproteinase; VAS: visual analog scale; mVAS: modified VAS; DMARD: disease-modifying antirheumatic drug; QTc: corrected electrocardiographic QT interval; HAQ: Health Assessment Questionnaire; MTX: methotrexate.

$$
\text { Personal non-commercial use only. The Journal of Rheumatology Copyright } \subset \text { }(2018 \text {. All rights reserved. }
$$


median had a mean baseline QTc of 436 ms compared with $412 \mathrm{~ms}$ for those without anti-CCP and SJC, CRP, and MMP-3 all below median levels $(p=0.003$; Figure 2$)$. Having any 1,2 , or 3 of these features was associated with progressively longer baseline QTc. Seven of the 22 RA patients with all 4 of the identified indicators of baseline QTc had a baseline QTc > $440 \mathrm{~ms}$ (32\%) compared with none of those with none of the indicators ( $\mathrm{p}=0.029$, data not shown).

$R A$ patients with the greatest reduction in CRP had the greatest reduction in QTc after TCZ treatment. Although a number of baseline characteristics were univariately associated with the change in QTc (Table 3, univariate models), only the change in CRP and the baseline QTc were significantly associated with the change in QTc when modeled together (Table 3 , reduced multivariable model). Together, these 2 characteristics along with age accounted for $44 \%$ of the explainable variability in the change in QTc (i.e., $\mathrm{R}^{2}=0.438 ; \mathrm{p}<0.001$ ). Interestingly, clinical responders did not have a greater change in QTc compared with nonresponders independent of the change in CRP or baseline QTc, even when more stringent definitions of response were

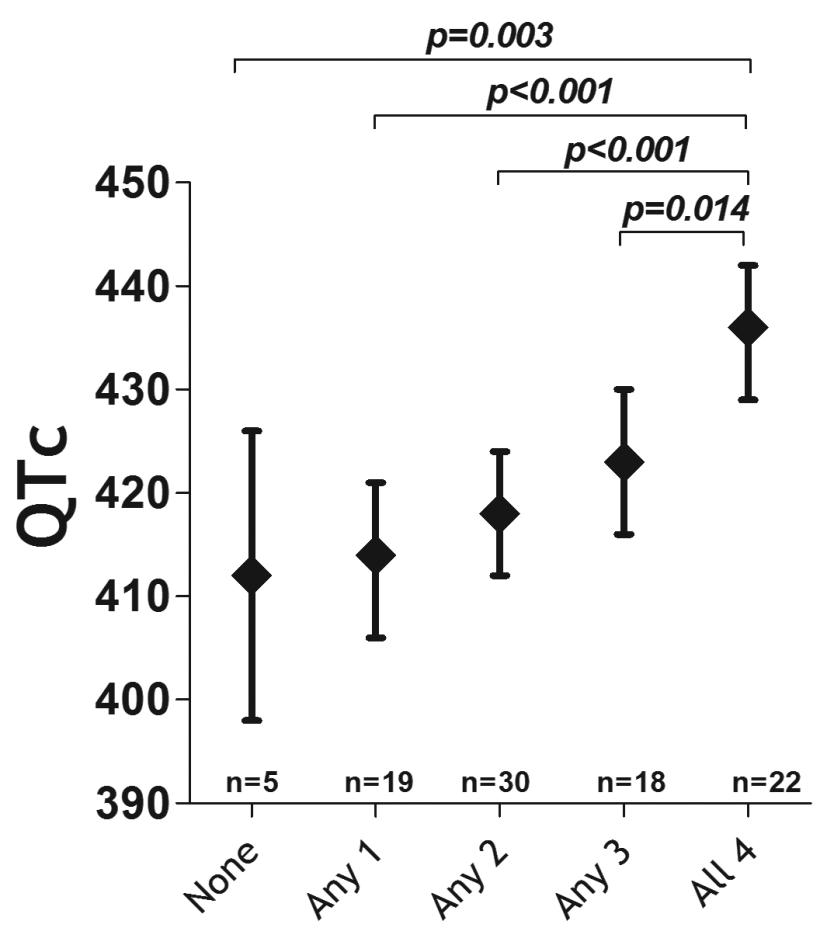

Features Present

Figure 2. Adjusted baseline QTc according to the number of features present. Means and $95 \% \mathrm{CI}$ are depicted. The features are the count of those identified from the reduced multivariable model depicted in Table 2. Presence for each feature was designated for anti-CCP $>20$ units, swollen joint count above the median of 4 joints, C-reactive protein greater than the median of $2.0 \mathrm{mg} / \mathrm{dl}$, and MMP-3 greater than the median of 150 units. Anti-CCP: anticyclic citrullinated peptide; MMP-3: matrix metalloproteinase 3; QTc: corrected electrocardiographic QT interval. applied (i.e., DAS remission or having a maximum of 1 swollen joint).

Patients with RA who were in the highest tertile of CRP reduction (a reduction of $\geq 4.0 \mathrm{mg} / \mathrm{dl}$ ) had a $26-\mathrm{ms}$ decrease in QTc, on average, compared with a 14-ms reduction among those in the tertile with the smallest reduction in CRP $(+0.09$ to $-1.0 \mathrm{mg} / \mathrm{dl}$; Figure $3 \mathrm{~A}$ ), a difference that was significant $(p=0.003)$. Those in the highest tertile of baseline QTc had a nearly 3-fold larger reduction in QTc after treatment compared with those in the lowest tertile of baseline QTc (Figure 3B). Interestingly, among those with a baseline QTc $\geq 440 \mathrm{~ms}$, each $1.0 \mathrm{mg} / \mathrm{dl}$ reduction in CRP was associated, on average, with a reduction in QTc of $11.2 \mathrm{~ms}(\mathrm{p}=0.002)$ compared with an average reduction in QTc of $3.4 \mathrm{~ms}$ for each $\mathrm{mg} / \mathrm{dl}$ reduction in CRP among those with a baseline QTC $<440 \mathrm{~ms}(\mathrm{p}=0.001 ; \mathrm{p}$ value for interaction $=0.039$; Figure 3C).

\section{DISCUSSION}

In this, to our knowledge, the largest study to date examining the effect of anticytokine therapy on QTc in RA, we observed a significantly higher QTc among a group of RA patients with a low prevalence of $\mathrm{CV}$ risk factors compared with a demographically matched group of non-RA controls. After 24 weeks of treatment with TCZ, QTc was reduced to levels that were significantly lower than those of the control group. The baseline QTc interval was strongly and independently associated with anti-CCP seropositivity, articular swelling, and several circulating inflammatory intermediates (CRP and MMP-3). However, only the change in CRP was a predictor of the change in QTc occurring with TCZ treatment. No other RA features were associated with the change in QTc independent of the change in CRP, including whether the patient had a clinical response to the drug. Interestingly, the association of the change in CRP with the change in QTc was markedly stronger among those with a prolonged baseline QTc.

Prolongation of the QTc interval is an established predictor of arrhythmia and sudden cardiac death in the general population, with moderate (QTc of $420-440 \mathrm{~ms}$ ) and extensive QTc prolongation (> $440 \mathrm{~ms}$ ) predictive of all-cause mortality ${ }^{13}$. Sudden cardiac death is also recognized as an important cause of death in patients with $\mathrm{RA}^{1}$; however, the pathophysiological mechanisms remain unclear. A strong link to systemic inflammation is suggested by several prior studies. Lazzerini, et al demonstrated a significant positive correlation between CRP and QTc duration in a cohort of 101 patients with chronic inflammatory arthritis ${ }^{6}$, and this association has been extended to circulating IL- 6 levels ${ }^{14}$ and other inflammatory cytokines in $\mathrm{RA}^{7}$. How elevated circulating inflammatory cytokines prolong QTc is unclear, because they may affect the myocardium directly, or indirectly by increasing central nervous system sympathetic drive on the heart ${ }^{1}$.

Personal non-commercial use only. The Journal of Rheumatology Copyright $\subset$ $\subset$ 2018. All rights reserved 
Table 3. Univariate and multivariable determinants of change in QTc.

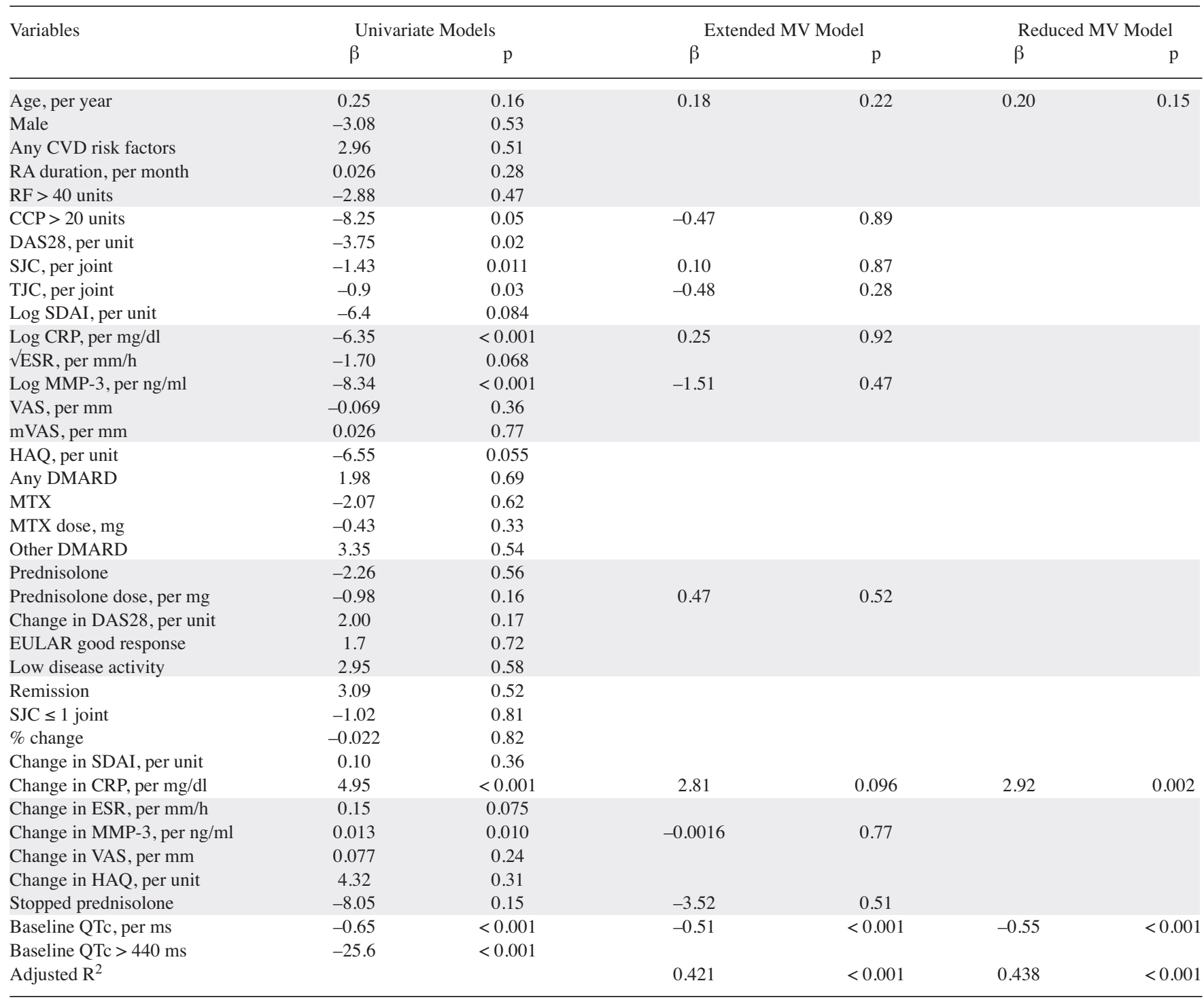

$\beta$ coefficients represent the average difference in the change in QTc interval per 1 unit higher of the indicated independent variable from linear regression modeling. The first column of univariate models represent individual models in which the indicated independent variable is the only covariate in the model. The MV models are single models containing each of the listed covariates. $\mathrm{R}^{2}$ represents the proportion of the variability of the change in QTc interval explained by the covariates in the model. MV: multivariable; RA: rheumatoid arthritis; CVD: cardiovascular disease; RF: rheumatoid factor; anti-CCP: anticyclic citrullinated peptide; DAS28: 28-joint count Disease Activity Score; SJC: swollen joint count; TJC: tender joint count; SDAI: Simplified Disease Activity Index; CRP: C-reactive protein; ESR: erythrocyte sedimentation rate; MMP: matrix metalloproteinase; VAS: visual analog scale; mVAS: modified VAS; DMARD: disease-modifying antirheumatic drug; QTc: corrected electrocardiographic QT interval; HAQ: Health Assessment Questionnaire; MTX: methotrexate; EULAR: European League Against Rheumatism.

Our study confirms these prior findings of a longer QTc associated with higher levels of circulating inflammatory markers, specifically CRP and MMP-3. We also found independent additive effects of SJC and seropositivity for anti-CCP on baseline QTc. Swollen joints are likely representative of additional unmeasured cytokines and inflammatory mediators, and indicative of additional inflammatory burden not determined by CRP or MMP-3. We also observed that anti-CCP-seropositive patients had longer QTc intervals. This could also represent a higher inflammatory burden in these patients, or there may be a direct link between antibodies against citrullinated proteins (ACPA) and the myocardium. In a prior publication ${ }^{15}$, we reported markedly higher staining for citrullinated proteins in the myocardial interstitium of patients with RA compared with non-RA controls and controls with systemic sclerosis and myocarditis. How ACPA may interact with the myocardium to prolong the QTc interval is unknown. However, the presence of anti-CCP did not impair the ability of TCZ to reduce QTc in our study after accounting for the change in CRP.

$$
\text { Personal non-commercial use only. The Journal of Rheumatology Copyright } \subset \text { }(2018 \text {. All rights reserved. }
$$




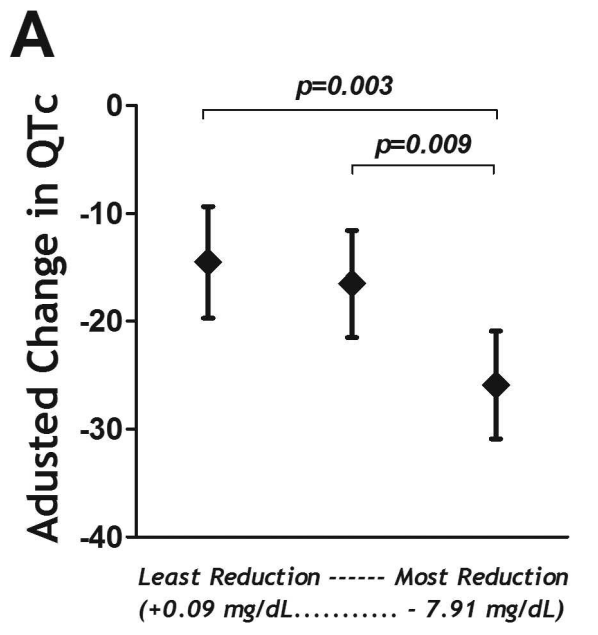

Tertiles of Change in CRP

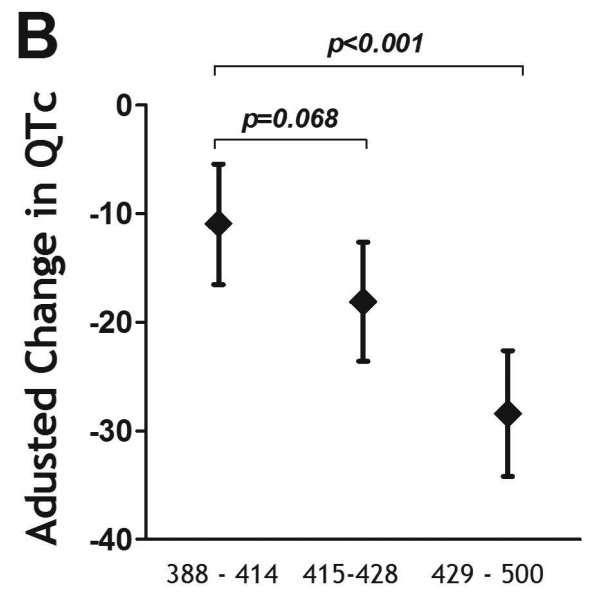

Tertiles of Baseline QTc (ms)

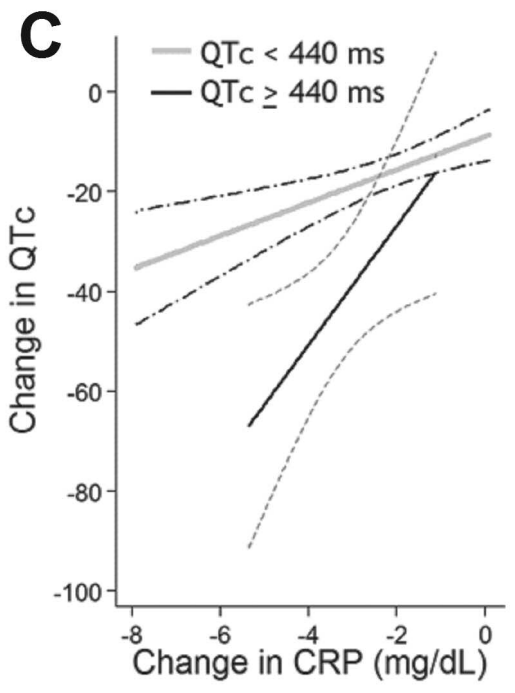

Figure 3. Adjusted change in QTc interval after tocilizumab treatment according to change in CRP and baseline QTc interval. Means and 95\% CI are depicted in panels A and B. Panel C depicts a more profound reduction in QTc according to the change in CRP among those with a higher baseline QTc interval ( $\mathrm{p}$ value for interaction $=0.039)$. QTc: corrected electrocardiographic QT interval; CRP: C-reactive protein.

Our study also lends additional credence to the concept of IL-6 as a driver of QTc prolongation in RA, because targeted inhibition of IL-6 was associated with a marked reduction in QTc to levels that were even lower than those in the non-RA healthy population controls. This could be through direct inhibition of IL-6, or indirectly through the ability of IL-6 inhibition to reduce other cytokines and inflammatory mediators in RA. Our study expands on the findings of a pilot study $^{8}$ in which a decrease in QTc was observed in 17 patients with RA after 12 weeks of TCZ treatment and was sustained to 24 weeks. Interestingly, this effect was independent of clinical response to TCZ, suggesting that the effect of TCZ on QTc is unrelated to its immunomodulating effect on synovitis. The ability of TCZ to reduce QTc to a level well below that found in healthy controls was somewhat unexpected. However, because the mechanism linking circulating IL-6 to QTc prolongation is likely not restricted to RA, it is possible, albeit speculative, that IL-6 inhibition in the general population may also have the effect of QTc reduction to the level we observed in the TCZ-treated patients with $\mathrm{RA}^{16}$.

The mechanism by which IL-6 inhibition reduced QTc is unclear. Previously, we reported that TCZ treatment significantly decreased left ventricular hypertrophy and normalized the aberrant left ventricular morphology that was associated with disease activity ${ }^{17}$. It is reasonable to posit that remodeling the myocardium to a more normalized structure may lead to QTc normalization. Additionally, an increasing body of evidence implicates macrophage-derived inflammatory cytokines, including tumor necrosis factor, IL-1, and IL-6, in electrophysiologic changes in cardiomyocytes, resulting in electrical remodeling of the heart that may predispose to prolonged QTc and ventricular arrhythmia (reviewed in
Lazzerini, et al $\left.^{18}\right)$. These include impaired potassium channel function ${ }^{19,20,21,22}$ and enhanced calcium channel function ${ }^{23,24}$ in cardiomyocytes, leading to prolongation of the cardiomyocyte action potential duration. Whether reduction of macrophage-derived cytokines with IL-6 inhibition is capable of reversing this electrical remodeling in RA warrants further study. Because the effect of TCZ on QTc was observed as early as 12 weeks in the pilot study by Lazzerini, et $a l^{8}$, it seems likely that the effect is mediated through electrical remodeling rather than structural remodeling, at least initially.

Our study has notable strengths and limitations. Among the strengths, the robust decline in QTc associated with decreasing CRP is a strong indicator of causality. However, without a comparison to untreated controls or a group treated with an immunomodulator targeting a different cytokine, we cannot assert that the observed normalization of QTc was not a natural phenomenon or that it is specific to IL-6 inhibition. In addition, IL-6 is the major cytokine determinant of hepatic CRP production. Thus, CRP may not be reflective of overall systemic inflammation in the setting of targeted IL-6 inhibition. While we focused on ECG as a marker of CVD, the ECG lacks specificity to detect early/preclinical myocardial lesions. We did not have additional imaging or advanced electrophysiological assessments to examine the mechanisms whereby IL-6 inhibition normalizes TCZ; however, based on our findings, such studies are certainly warranted and could prove useful not only to the study of arrhythmia in RA, but also for the general population. Finally, medications known to prolong QTc were not part of our data collection. However, for these to have impugned the differences detected they would have to have been markedly unbalanced between the RA and control groups and changed after TCZ treatment among enough patients with RA to normalize

Personal non-commercial use only. The Journal of Rheumatology Copyright @ 2018 . All rights reserved. 
the QTc of the entire group. Such a scenario is unlikely to account for the magnitude of the differences and changes detected.

The marked normalization of QTc observed with TCZ treatment, and its close parallel with CRP reduction, support the premise that systemic inflammation contributes to cardiac repolarization abnormalities in RA that may be amenable to treatment, and suggest an antiarrhythmic effect of IL-6 inhibition that may be beneficial for patients with RA. Our data provide further evidence of the close link between RA autoimmunity, inflammation, and cardiac repolarization that may contribute to the known higher CVD risk in this population. Further investigation is warranted to evaluate the effect of TCZ on arrhythmia and sudden cardiac death in RA.

\section{ACKNOWLEDGMENT}

The authors thank all the clinical engineers, and the hematology and rheumatology staff at Nihon University School of Medicine Hospital for their assistance.

\section{REFERENCES}

1. Maradit-Kremers H, Crowson CS, Nicola PJ, Ballman KV, Roger VL, Jacobsen SJ, et al. Increased unrecognized coronary heart disease and sudden deaths in rheumatoid arthritis: a population-based cohort study. Arthritis Rheum 2005;52:402-11.

2. Huikuri HV, Castellanos A, Myerburg RJ. Sudden death due to cardiac arrhythmias. N Engl J Med 2001;345:1473-82.

3. Montanez A, Ruskin JN, Hebert PR, Lamas GA, Hennekens CH. Prolonged QTc interval and risks of total and cardiovascular mortality and sudden death in the general population: a review and qualitative overview of the prospective cohort studies. Arch Intern Med 2004;164:943-8.

4. Chauhan K, Ackerman MJ, Crowson CS, Matteson EL, Gabriel SE. Population-based study of QT interval prolongation in patients with rheumatoid arthritis. Clin Exp Rheumatol 2015;33:84-9.

5. Panoulas VF, Toms TE, Douglas KM, Sandoo A, Metsios GS, Stavropoulos-Kalinoglou A, et al. Prolonged QTc interval predicts all-cause mortality in patients with rheumatoid arthritis: an association driven by high inflammatory burden. Rheumatology 2014;53:131-7.

6. Lazzerini PE, Acampa M, Capecchi PL, Hammoud M, Maffei S, Bisogno S, et al. Association between high sensitivity C-reactive protein, heart rate variability and corrected QT interval in patients with chronic inflammatory arthritis. Eur J Int Med 2013;24:368-74.

7. Adlan AM, Panoulas VF, Smith JP, Fisher JP, Kitas GD. Association between corrected QT interval and inflammatory cytokines in rheumatoid arthritis. J Rheumatol 2015;42:421-8.

8. Lazzerini PE, Acampa M, Capecchi PL, Fineschi I, Selvi E, Moscadelli V, et al. Antiarrhythmic potential of anticytokine therapy in rheumatoid arthritis: tocilizumab reduces corrected QT interval by controlling systemic inflammation. Arthritis Care Res 2015;67:332-9.

9. Aletaha D, Neogi T, Silman AJ, Funovits J, Felson DT, Bingham CO 3rd, et al. 2010 rheumatoid arthritis classification criteria: an American College of Rheumatology/European League Against Rheumatism collaborative initiative. Arthritis Rheum 2010; 62:2569-81
10. Prevoo ML, van 't Hof MA, Kuper HH, van Leeuwen MA, van de Putte LB, van Riel PL. Modified disease activity scores that include twenty-eight-joint counts. Development and validation in a prospective longitudinal study of patients with rheumatoid arthritis. Arthritis Rheum 1995;38:44-8.

11. Fransen J, van Riel PL. The Disease Activity Score and the EULAR response criteria. Rheum Dis Clin North Am 2009;35:745-57.

12. Algra A, Tijssen JG, Roelandt JR, Pool J, Lubsen J. QTc prolongation measured by standard 12-lead electrocardiography is an independent risk factor for sudden death due to cardiac arrest. Circulation 1991;83:1888-94.

13. Straus SM, Kors JA, De Bruin ML, van der Hooft CS, Hofman A, Heeringa $\mathrm{J}$, et al. Prolonged QTc interval and risk of sudden cardiac death in a population of older adults. J Am Coll Cardiol 2006;47:362-7

14. Lazzerini PE, Capecchi PL, Bertolozzi I, Morozzi G, Lorenzini S, Simpatico A, et al. Marked QTc prolongation and torsades de pointes in patients with chronic inflammatory arthritis. Front Cardiovasc Med 2016;3:31.

15. Giles JT, Fert-Bober J, Park JK, Bingham CO 3rd, Andrade F, Fox-Talbot K, et al. Myocardial citrullination in rheumatoid arthritis: a correlative histopathologic study. Arthritis Res Ther 2012;14:R39.

16. Lazzerini PE, Laghi-Pasini F, Bertolozzi I, Morozzi G, Lorenzini S, Simpatico A, et al. Systemic inflammation as a novel QT-prolonging risk factor in patients with torsades de pointes. Heart 2017;103:1821-9.

17. Kobayashi H, Kobayashi Y, Giles JT, Yoneyama K, Nakajima Y, Takei M. Tocilizumab treatment increases left ventricular ejection fraction and decreases left ventricular mass index in patients with rheumatoid arthritis without cardiac symptoms: assessed using 3.0 tesla cardiac magnetic resonance imaging. J Rheumatol 2014;41:1916-21.

18. Lazzerini PE, Capecchi PL, Laghi-Pasini F. Systemic inflammation and arrhythmic risk: lessons from rheumatoid arthritis. Eur Heart J 2017;38:1717-27.

19. Fernandez-Velasco M, Ruiz-Hurtado G, Hurtado O, Moro MA, Delgado C. TNF-alpha downregulates transient outward potassium current in rat ventricular myocytes through iNOS overexpression and oxidant species generation. Am J Physiol Heart Circ Physiol 2007;293:H238-45.

20. Petkova-Kirova PS, Gursoy E, Mehdi H, McTiernan CF, London B, Salama G. Electrical remodeling of cardiac myocytes from mice with heart failure due to the overexpression of tumor necrosis factor-alpha. Am J Physiol Heart Circ Physiol 2006;290:H2098-107.

21. Wang J, Wang H, Zhang Y, Gao H, Nattel S, Wang Z. Impairment of HERG $\mathrm{K}(+)$ channel function by tumor necrosis factor-alpha: role of reactive oxygen species as a mediator. J Biol Chem 2004;279:13289-92.

22. Monnerat G, Alarcón ML, Vasconcellos LR, Hochman-Mendez C, Brasil G, Bassani RA, et al. Macrophage-dependent IL-1 $\beta$ production induces cardiac arrhythmias in diabetic mice. Nature Com 2016;7:13344.

23. Hagiwara Y, Miyoshi S, Fukuda K, Nishiyama N, Ikegami Y, Tanimoto K, et al. SHP2-mediated signaling cascade through gp130 is essential for LIF-dependent I CaL, [Ca2+]i transient, and APD increase in cardiomyocytes. J Mol Cell Cardiol 2007;43:710-6.

24. Li YH, Rozanski GJ. Effects of human recombinant interleukin-1 on electrical properties of guinea pig ventricular cells. Cardiovasc Res 1993;27:525-30. 